\title{
BARRERAS EN EL DESARROLLO PROFESIONAL FEMENINO*
}

\author{
BARRIERS TO CAREER DEVELOPMENT OF WOMEN
}

\author{
Magdalena Suárez Ortega** \\ $U N E D$
}

\begin{abstract}
RESUMEN
En este trabajo se analizan las barreras percibidas por un grupo de mujeres que quieren incorporarse al empleo desde la formación ocupacional. Los resultados encontrados ponen de manifiesto cómo aspectos personales, académico-formativos, profesionales y contextuales se asocian a estereotipos de género los cuales son sentidos por las protagonistas como factores que obstaculizan su formación e inserción laboral. Conocer estas barreras y comprender su proceso de gestación favorecerá su eliminación de la oferta formativa y orientadora, con la que cuentan actualmente las mujeres que quieren capacitarse profesionalmente.
\end{abstract}

Palabras clave: barreras relativas a la carrera, género, desarrollo profesional femenino, formación profesional ocupacional, inserción laboral, subjetividad, voz.

\begin{abstract}
In this paper we analyze the barriers perceived by a group of adult women with low qualification, who want to get into the labour market from the occupational vocational training. The results show how personal, academic-formative, professional and contextual aspects, that characterize the life project of women, are associated to gender stereotypes. Women felt these factors like barriers or obstacles to their access to job and to the fullfiment of their professional projects. Being aware of these barriers will help us to improve this training process to remove gender obstacle.
\end{abstract}

Keywords: career related barriers, gender, professional development of women, occupational vocational training, labour insertion, subjectivity, voice.

\footnotetext{
* Este artículo es un trabajo parcial de mi tesis doctoral, titulada La construcción del proyecto profesional-vital de mujeres adultas: un reto para la intervención orientadora. Universidad de Sevilla, 2006. Dirigida por las profesoras: $\mathrm{M}^{\mathrm{a}}$ Soledad García Gómez y $\mathrm{M}^{\mathrm{a}}$ Teresa Padilla Carmona pertenecientes, respectivamente, a los Departamentos de Didáctica y Organización Educativa, y Métodos de Investigación y Diagnóstico en Educación, de la Universidad de Sevilla. Dicha Tesis ha sido premiada por el Consejo Andaluz de Relaciones Laborales, Consejería de Empleo, Junta de Andalucía, en 2007.

** Contacto: Universidad Nacional de Educación a Distancia, Departamento de Métodos de Investigación y Diagnostico en Educación I. Facultad de Educación, Madrid, España. E-mail: msuarez@edu.uned.es
} 


\section{Introducción}

El término barreras profesionales o barreras relativas a la carrera (career related barriers) ha sido definido como "eventos o condiciones, ya sean de la persona o de su ambiente, que dificultan el proceso de desarrollo profesional" (Swanson y Woitke, 1997: 446). Con ello se alude a ciertos factores internos de la persona, así como a otros externos, relativos al contexto o medio en que esta se inserta.

Los trabajos existentes señalan algunos tipos de barreras, dando como resultado diferentes clasificaciones. Harmon (1977), Farmer (1985; 1997), Swanson y Woitke (1997) se han referido a barreras internas y externas, Swanson y Tokan (1991) a barreras sociales-interpersonales, actitudinales, e interaccionales, Phillips y Imhoff, (1997), Russel y Burguess (1998), a barreras anteriores y posteriores a la inserción profesional, o Fiztgerald y Weitzman (1992), y Melamed (1995), que aluden a barreras debidas a la interacción de factores en el desarrollo profesional.

Teniendo en consideración las clasificaciones anteriores, y entendiendo la carrera como un proceso multidimensional en el que se incluyen múltiples aspectos, las barreras son interactivas, cambiantes y que pueden manifestarse en sentidos diversos. Si atendemos a la falta de cualificación profesional como barrera relativa a la carrera, pueden estar implicados factores como la edad, el sexo, la discriminación por razón de género (oportunidades educativas y laborales), el ambiente, o las experiencias previas.

Algunos trabajos han señalado la influencia de diversos factores en el proceso de decisión vocacional. Sebastián et al. (2005) evidencian la importancia de un currículum oculto en la escuela, por el que se siguen dando y reproduciendo estereotipos sexistas. Lozano y Repetto (2007), por su parte, hacen hincapié en la relación del género, el nivel educativo y los intereses profesionales. En este sentido nos parece relevante el trabajo de Sánchez García (2004), quien resalta la interactividad de factores en la carrera. De forma específica para el trabajo que nos ocupa, esta autora pone de manifiesto que la edad, la cualificación y el género son factores determinantes que condicionan el acceso al empleo, al mismo tiempo que importantes factores de exclusión, que se agudizan en grupos con menores recursos.

Uno de estos grupos son las mujeres adultas con escasa cualificación que quieren acceder al empleo desde la formación ocupacional. Son mujeres que han contado con escasas oportunidades educativas y profesionales, si nos situamos en sus condiciones vitales y contextuales de partida (escaso nivel económico, entornos rurales, y desarrollo de sus trayectorias de vida en torno a los años cincuenta-sesenta en Andalucía). Por ello, aunque autores como Cavanaugh y Blanchard-Fields (2002) ponen de relieve algunas barreras de género comunes como el techo de cristal, el conflicto de roles, la segregación profesional o la violencia, el colectivo citado, en nuestra opinión, merece una atención especial, ya que los procesos de re-inserción femeninos en la adultez adoptan características diversas con respecto a otros momentos, dándose en esta etapa un importante incremento de la experiencia vital y/o manifestándose nuevas necesidades formativas/profesionales como fruto de unas determinadas oportunidades o posibilidades previas de desarrollo. En este sentido, Coria, Freixas y Covas (2005: 28) aluden a tres aspectos fundamentales que intervienen en las transiciones femeninas:

"El primero es la conformación de una subjetividad femenina construida como "satélite del deseo ajeno". El segundo es la dependencia afectiva, económica y legal que aún sigue vigente 
y fuertemente arraigada en el psiquismo de no pocas mujeres (...). El tercero es el modelo de maternidad que, además de ser concebido como incondicional, abnegado y altruista, se plantea para las mujeres como un rol vitalicio ejercido de por vida".

En un trabajo anterior (Suárez, 2004) analizamos las dificultades y limitaciones que tienen las mujeres adultas que quieren incorporarse al mercado laboral. Los resultados apuntan a un aumento de obstáculos y a una disminución de las oportunidades de empleabilidad. Los desfases son mayores en las mujeres que se han vinculado exclusivamente al ámbito doméstico y que han estado desvinculadas de la formación, disminuyendo las posibilidades de inserción con el paso del tiempo. En este contexto, Montané (1993) alude a las menores posibilidades de empleabilidad de las amas de casa sin experiencia profesional. Algunas de las dificultades que señalan van en la línea de escasa iniciativa, baja autoestima, escasa o desfasada cualificación, así como la insuficiente disponibilidad real para el empleo.

Por su parte, Gimeno y Rocabert (1998) destacan que los múltiples roles y el conflicto de rol tiene importantes consecuencias cuando las mujeres quieren acceder al empleo, lo que para Padilla (2002) se convierte en la principal barrera a la que se enfrentan las mujeres adultas que quieren acceder al mercado laboral. Blanch (1992) señala la complejidad que entraña este proceso de transición de la mujer del estatus de ama de casa al de trabajadora ocupada, que pasa por la reformulación de los itinerarios educativos o académicos. En él intervienen factores que lo hacen especialmente difícil, ya que no sólo tienen que superar carencias formativas o las relativas a los múltiples roles, sino que también las propias estructuras y fuerzas laborales ofrecen resistencia.

En este proceso de búsqueda de empleo muchas mujeres no tienen la disponibilidad suficiente para mantener la actividad que conlleva dicha situación. En esta disponibilidad laboral, según Poal (1993), intervienen dos dimensiones importantes de la persona. Por un lado, una dimensión objetiva constituida por factores, como disponibilidad real de tiempo, necesidad económica, nivel de conocimiento de las características y demandas del mercado laboral. Por otro, una dimensión subjetiva donde intervienen aspectos como el estilo de búsqueda de empleo, el valor atribuido al trabajo, las expectativas con respecto al mismo, la claridad en los valores y metas laborales, o la prioridad dada a la propia autorrealización.

\section{Método}

\section{El objeto de estudio}

Nos centramos en las mujeres adultas con escasa cualificación de la provincia de Sevilla que quieren acceder al empleo a edad adulta, con la intención de conocer las barreras que perciben en el proceso de formación para el empleo en el que están inmersas. Son mujeres que tienen una media de edad en torno a los cuarenta años, son madres y están, en su mayoría, casadas, dedicándose principalmente a la familia.

Estas mujeres comienzan a inscribirse como paradas en el Servicio Andaluz de Empleo, estando sus perfiles como demandantes caracterizados por unos tiempos prolongados de alejamiento de la formación y del mercado laboral denominado "formal". Estos cambios en las mujeres (acceder a la formación para el empleo), se producen en interacción con los momentos personales y familiares que experimentan, los contextos en los que se sitúan, las 
nuevas demandas que plantean y las posibles respuestas que dan a las mismas, así como con la sociedad en la que viven y han vivido.

\section{Las participantes y sus contextos vitales}

La muestra seleccionada responde a criterios estructurales, es intencional, atendiendo al tipo social: en este caso, mujeres cuyas vidas las han dedicado durante un tiempo considerable al cuidado de la familia y del hogar, manifestando en la actualidad deseos o inquietudes frente al empleo. Son mujeres que participan en los momentos iniciales del estudio en alguna actividad formativa con carácter ocupacional, y que pertenecen a diferentes contextos locales-rurales de la provincia de Sevilla.

En esta investigación han participado un total de 40 mujeres, aunque su implicación, adquiere diferencias notables según las fases. En la primera fase (extensiva) intervienen la totalidad de mujeres (cuarenta), y en la segunda (intensiva) se procede a la selección de ocho de ellas. En este trabajo nos centramos en la segunda fase de la investigación, participando en el trabajo de campo ocho informantes, dos por cada uno de los contextos citados.

Las mujeres pertenecen a cuatro municipios sevillanos de las comarcas de la Sierra Norte, la Campiña, el Área metropolitana y el Aljarafe. Los pueblos a los que pertenecen las mujeres, aunque divergen fundamentalmente en su amplitud, recursos, distancia con respecto a la capital y nivel experimentado de desarrollo en los últimos años, tienen en común su carácter local/rural, la alta demanda de empleo, concretamente femenino en tramos de edad adulta, el medio fundamental de vida sobre el que intentan desarrollarse (la agricultura y/o la ganadería), y la escasez de ofertas de empleo.

\section{Metodología y proceso de investigación}

La metodología utilizada es cualitativa, permitiéndonos un acercamiento al objeto de estudio desde una perspectiva interna (Curtis, 1978; Taylor y Bogdan, 1986). Es decir, éste se explora desde la perspectiva de quienes lo viven y desde las formas en que lo perciben, sienten y experimentan, poniendo el acento en el significado subjetivo, en las voces y propias experiencias de las mujeres.

La investigación se lleva a cabo desde 2002-2003 hasta 2005-2006. Siguiendo a Rodríguez, Gil y García (1999), las fases generales fueron: preparación, trabajo de campo, análisis e información. Este proceso ha sido abierto, flexible y emergente, configurándose a medida que avanzaba el estudio. De forma específica, la primera fase adquiere un carácter extensivo y la segunda, en la que nos centramos, intensivo, de profundización sobre el objeto de estudio.

\section{Técnicas para la recogida y el análisis de la información}

Como técnicas de recogida de datos utilizamos el relato de vida y las notas de campo. Para proceder a la aplicación de los relatos (técnica central de recogida de datos), de las cuarenta mujeres que intervienen en la primera fase, pertenecientes a las cuatro zonas sevi- 
llanas mencionadas, se seleccionan ocho, dos mujeres de cada uno de los contextos implicados, desarrollándose un total de ocho relatos.

Dicha selección se realizó teniendo como base el conocimiento que aportado por la primera fase, y los factores que daban muestra de la diversidad de perfiles encontrados (la edad de las mujeres, las situaciones laborales -empleo/desempleo- y las personales - estado civil o situación de convivencia-, la implicación en actividades socio-formativas, la preocupación con respecto a sus situaciones vitales, y el contexto).

Los relatos de vida se desarrollaron sobre un ciclo de entrevistas en profundidad. Para ello, se utilizaron como apoyo unos guiones de preguntas, que se adecuaron en torno a los fines de la investigación y se estructuraron conforme a las etapas biográficas. En el cuadro 1 presentamos los objetivos de dichos guiones en cada una de las etapas.

CUADRO 1: Extracto de los guiones de preguntas utilizados para la recogida de datos.

\begin{tabular}{|c|}
\hline ETAPAS BIOGRÁFICAS \\
\hline $\begin{array}{l}\text { 1. PRIMERA ETAPA: ESBOZO BIOGRÁFICO GENERAL } \\
\text { Contenido a explorar: Trayectorias vitales de las mujeres, experiencias previas asociadas al ámbi- } \\
\text { to personal, académico-formativo y profesional. }\end{array}$ \\
\hline $\begin{array}{l}\text { 2. SEGUNDA ETAPA: PROFUNDIZACIÓN (TRANSICIONES) } \\
\text { Contenido a explorar: Exploración de las situaciones actuales: el acceso y el desarrollo de las ac- } \\
\text { tivides de formación profesional ocupacional. Vinculación con los contenidos anteriores. } \\
\text { 3. TERCERA ETAPA: PROFUNDIZACIÓN (PROYECTO PROFESIONAL) } \\
\text { futuras y planificación profesional. Vinculación con los contenidos anteriores. }\end{array}$ \\
\hline
\end{tabular}

Tras la recogida de datos, las tareas centrales de análisis, siguiendo a Miles y Huberman (1994), han sido: transcripción y reducción de los mismos (codificación y categorización), disposición, organización e interpretación de la información, previa codificación ("sistema de indización") y elaboración de un sistema jerárquico de categorías, y obtención de conclusiones. En el cuadro 2 presentamos un extracto del sistema de categorías, así como de la definición y ejemplificación de su contenido.

El informe de resultados ha sido organizado atendiendo al sistema de categorías. No obstante, se ha utilizado la triangulación de técnicas y la comparación entre casos. Ello facilitó la obtención de información común, en la que existía acuerdo, y otra más concreta, que aportaban casos aislados. Los datos que se presentan a continuación están basados en las opiniones mayoritarias que han dado las mujeres, utilizándose nombres ficticios para guardar el anonimato.

\section{Resultados}

\section{La edad de las mujeres y la pareja}

En los discursos emitidos puede apreciarse cómo la edad es sentida como una barrera importante para el acceso al empleo. Debido a la edad, ellas sienten que entran en un círcu- 
CUADRO 2: Extracto del sistema de categorías, de su proceso de definición y ejemplificación.

\begin{tabular}{|c|}
\hline SISTEMA DE CATEGORÍAS \\
\hline DIMENSIÓN: Barreras relativas a la carrera \\
\hline DEFINICIÓN: Limitaciones o dificultades en el desarrollo profesional femenino \\
\hline La edad de acceso a la formacial empleo. \\
\hline El matrimonio y la pareja. \\
\hline La maternidad, las responsabilidades familiares. \\
\hline La escasa formación reglada y la baja cualificación profesional. \\
\hline Los escasos recursos económicos. \\
\hline La limitada oferta formativa y laboral en los pueblos. \\
\hline Las bajas expectativas de éxito y autoestima. \\
\hline PROCESO DE DEFINICIÓN Y EJEMPLIFICACIÓN DE LAS CATEGORÍAS \\
\hline Subcategoría: Conflicto de roles Código: ROLE. \\
\hline Definición: Efecto multiplicador de roles familiares-laborales. \\
\hline $\begin{array}{l}\text { Ejemplo: Salía a las ocho de la mañana para estar el día aquí y luego me iba por la noche, a las } \\
\text { diez de la noche, y no venía hasta las tres de la tarde a mi casa, luego ese día descansaba, me iba } \\
\text { otra vez a las ocho de la mañana y venía, echaba todo el día y toda la noche, o sea, que he estado } \\
\text { cuidando enfermos, he estado trabajando en el hospital, en un bar [Relato de vida]. }\end{array}$ \\
\hline Subcategoría: Nivel económico Código: ECON. \\
\hline Definición: Independencia económica de las mujeres. \\
\hline $\begin{array}{l}\text { Ejemplo: Algo tan simple como tener dinero para ti, para poder formarte, o moverte para buscar tra- } \\
\text { bajo, fotocopiar tu currículum, poder comprarte un ordenador. Para estudiar se necesita dinero, } \\
\text { además de tiempo, dinero [Notas]. }\end{array}$ \\
\hline
\end{tabular}

lo vicioso, que se complica cada vez más: pasan los años, sus condiciones de trabajo se mantienen y sus situaciones de precariedad laboral se acentúan.

¿Qué nos queda a nosotros? ¿Qué queda para las mujeres de nuestra edad y que pasan por estos problemas? Al final limpiar o cuidar a ancianos, es lo único [Notas, Dolores].

Pocas posibilidades, por la edad lo primero, que eso es lo más difícil que yo veo, lo que más influye ahora mismo esla edad, aunque ahora, por lo visto, también han sacado cosas que están dando trabajo a personas mayores de treinta y cinco años [Rosa, relato de vida].

Las mujeres adoptan un papel de sumisión al marido, que puede explicarse desde la dependencia económica, que les impide una vida propia, así como desde una dependencia psicológica que afecta a la toma de decisiones. Me condicioné mucho a mi marido, si tenía que salir salía con él, si no iba con él no salía, hasta que ya conocí a otras madres del co- 
legio, a otras mujeres, por eso decía él que me abrieron los ojos en el colegio [Aurora, relato de vida]. Si yo digo de hacer algo al momento: “idéjate!”, “ ¿qué falta te hace?”, es lo primero que me dice mi marido, “qué falta te hace?”, “'tú tienes necesidad de trabajar?" [Pilar, relato de vida].

\section{La maternidad y la difícil conciliación familiar-laboral}

La maternidad es sentida como una barrera importante para el desarrollo profesional femenino, ya que aumentan las responsabilidades familiares a las que estas mujeres hacen frente. Esta situación se complica a medida que aumenta el número de hijos e hijas, y cuentan con otras personas a su cargo. Es complicado porque además de tus hijos, luego te encuentras con otras personas, en mi caso, yo soy la que me encargo de cuidar a mi madre y a mi hermano, entonces es no terminar nunca, y por supuesto no tener nada de tiempo para ti [Notas, Aurora].

Las mujeres sienten dificultades para desarrollarse profesionalmente desde unos modelos discriminatorios de familia. Para ellas, compaginar trabajo fuera y dentro de la casa implica aumentar la jornada laboral, así como impedirles contar con opciones laborales más óptimas o con posibilidades de aumentar su cualificación profesional. El tiempo familiar más el tiempo dedicado al trabajo fuera de casa supone casi toda la jornada, quedando un intervalo temporal mínimo en el que resulta compleja la búsqueda de empleo o la formación.

Salía a las ocho de la mañana para estar el día aqui y luego me iba por la noche, a las diez de la noche, y no venía hasta las tres de la tarde a mi casa, luego ese día descansaba, me iba otra vez a las ocho de la mañana y venía, echaba todo el día y toda la noche, o sea, que he estado cuidando enfermos, he estado trabajando en el hospital, en un bar [Rocío, relato de vida].

\section{El nivel educativo, la escasa cualificación laboral y las dificultades de acreditación}

Las mujeres también resaltan la dificultad que conlleva afrontar la búsqueda de empleo desde niveles básicos, mínimos, de educación reglada. Asociado a esta barrera, que se explica desde las oportunidades y posibilidades que tuvieron en su etapa de escolarización (sobre años cincuenta-sesenta), ellas plantean también la dificultad de acreditación de este nivel educativo. Esto es algo que complica la inserción formativa y laboral de las mujeres, en la medida que no cuentan con el documento que acredite que poseen estos niveles formativos. Por tanto, se encuentran indefensas frente a las ofertas de empleo, incluso de formación, en las que tienen que acreditar sus niveles de educación reglada.

Si te das cuenta no tengo nada, ningún documento que acredite nada de lo que yo pueda hacer y es que yo creo que es la pescadilla que se muerde la cola, porque ya te digo, yo estoy en un bar porque yo sé hacer ese trabajo y lo acreditaría con la experiencia que tengo, pero que tampoco puedo acreditarlo porque no he estado asegurada, asi es dificil encontrar trabajo, muy dificil [Carmen, relato de vida].

Es dificil poder trabajar porque no tienes experiencia, pero si no trabajas nunca la tienes, $y$ estamos siempre en el mismo punto, no trabajas porque te falta experiencia, y si nunca te contratan nunca puedes justificarla [Rosa, Notas]. 


\section{El difícil acceso a las ofertas formativas y las vías de selección}

Se han puesto de relieve, no obstante, dificultades para acceder a las ofertas formativas existentes asociadas a la cercanía o lejanía de los recursos de que disponen. No todas las mujeres pueden acceder a una actividad formativa, y su acceso depende de aspectos como el horario o la duración. Si a esto se le une que han de desplazarse a otros pueblos o la capital para desarrollarla, las mujeres terminan por no acceder a ellas.

Es que al ser un pueblo muy pequeño no hay muchas posibilidades de formación, como no sea la escuela taller esa, porque aqui no hay instituto y si no hay instituto pues no te puedes formar a gran escala. No hay habitantes y si no hay habitantes... si los niños de tercero y cuarto de ESO se tienen que ir fuera, tú date cuenta, pues si hubiera aqui un instituto ipues más gente estudiaría! (...) Por coste económico también [Dolores, relato de vida].

Las mujeres también reflejan procesos de selección ocultos en los municipios, sin criterios claros, que propician el favoritismo, tanto en las ofertas de empleo como en las formativas. Desde estos aspectos resaltan criterios confusos en los cursos, falta de información en las entrevistas de trabajo, guiándose más por aspectos subjetivos que objetivos en relación con el puesto. Muchas veces me he enterado de cursos que me han interesado cuando ya estaba el cupo cerrado, me he enterado por la gente que ya estaba dentro, entonces vas y es para nada, ya no puedes entrar [Notas, Milagros].

\section{Las escasas ayudas al empleo femenino y la falta de recursos económicos propios}

Las mujeres hacen referencia a unas escasas ayudas sociales de apoyo al empleo femenino, en relación con la infraestructura de los pueblos. Aluden, sobre todo, a la "creación de guarderías y comedores" municipales, a la generación de posibilidades relacionadas con el ocio y tiempo libre de los y las jóvenes, así como con recursos físicos, locales, o herramientas que le permitan ensayar alternativas laborales.

Las familias en las que se sitúan las mujeres cuentan con niveles económicos bajos. Los maridos trabajan en el campo, en la construcción o en industrias, o algunos de ellos se encuentran en el paro. Las mujeres no cuentan con dinero propio que puedan invertir en la formación o en la búsqueda de empleo. Esta situación se complica aún más en mujeres separadas o divorciadas, que se encargan de mantener a la familia. El acceso a empleos en condiciones precarias y contar con una única entrada económica en la familia es algo que dificulta aún más sus posibilidades de mejora profesional/vital.

Algo tan simple como tener dinero para ti, para poder formarte, o moverte para buscar trabajo, fotocopiar tu curriculum, poder comprarte un ordenador. Para estudiar se necesita dinero, además de tiempo, dinero [Rosa, Notas].

\section{La anticipación negativa a los cambios y las bajas expectativas de éxito}

Las mujeres sienten la anticipación negativa a los cambios como limitación para su desarrollo profesional. Esta barrera es sentida desde la propia valoración sobre sus posibili- 
dades de mejora pensando en otras personas. Anticipan un escaso apoyo por parte de las parejas, principalmente, y de sus hijos, sobre todo los mayores y varones, sintiendo la negación ante la posibilidad de iniciar una nueva trayectoria laboral.

Si yo dijera: pues empiezo en serio a buscar trabajo me supondría una pelea con mi marido, porque no quiere que haga nada (...), me supondría ya ves, que me den como más libertad [Pilar, relato de vida].

Cada vez que me sale un trabajo o me ilusiono con algo encuentro problemas en mi casa, ya mi marido empieza a decirme que por qué me agobio, que no tengo ninguna necesidad de estar así, que no hace falta dinero en casa y que podemos arreglarnos con lo que tenemos [Carmen, Notas].

Otro de los aspectos que complican las vidas de las mujeres son sus bajas expectativas de éxito. La limitación en este caso se atribuye a sí mismas, a la falta de capacidad, de conocimientos, y de habilidades sentidas frente a la mejora profesional. Ello hace que experimenten una baja autoestima y una escasa autoeficacia frente a la mejora de sus situaciones laborales. Según ellas no cuentan con recursos suficientes para acceder a un empleo, mostrando sentimientos de impotencia para el cambio. Si hubiera estudiado ahora tendría trabajo [Carmen, Notas]. No debería de haber dejado mi trabajo en aquel tiempo, ahora es imposible tenerlo [Pilar, Notas].

Las mujeres sienten un temor atribuido a su falta de habilidad, conocimientos, actitudes o recursos. Para ellas es complejo vivir en la incertidumbre y lanzarse a aceptar un empleo en el que no saben qué tienen que hacer, ni si serán capaces de ello. Esto complica las escasas posibilidades que estas mujeres tienen de acceder al empleo.

\section{Conclusiones}

Las mujeres que participan en esta investigación, mujeres con escasa cualificación, que viven en pueblos de distintas comarcas sevillanas, y que quieren acceder al empleo desde la formación ocupacional habiéndose dedicado durante muchos años en exclusividad a la familia, destacan una serie de barreras relativas a su desarrollo profesional, que median en sus posibilidades de reorientación profesional. Ellas sienten que la edad con la que se enfrentan al mercado laboral es un impedimento importante para encontrar trabajo.

A esta barrera se vinculan las propias situaciones personales, formativas y profesionales, mediatizadas por los procesos de socialización sesgados por razón de género en los que han sido educadas y en los que viven. Así, se aprecia cómo algunas parejas influyen en las decisiones de las mujeres, con el matrimonio aumentan las responsabilidades familiares, y la maternidad es sentida como limitación, concluyéndose que el número de hijos e hijas que ellas tienen es decisivo en cómo afrontan y pueden afrontar su desarrollo profesional. También influyen negativamente en dicho proceso las cargas familiares con las que cuentan, personas dependientes o que tienen a su cargo. Esto se asocia a las situaciones económicas de las mujeres, al mismo tiempo que las propias atribuciones sobre estos roles; son ellas quienes tradicionalmente se han encargado de estos cuidados y sobre quienes sienten que recaen, sin implicación de otros miembros de la familia y sin la posibilidad de utilizar recursos o vías alternativas. 
Puede concluirse, asimismo, un aumento de responsabilidades en las mujeres que influye en la disponibilidad real para el empleo. En este sentido, las mujeres han manifestado una barrera fundamental como es el disponer de tiempos propios, para sí, para la formación y para el empleo, la búsqueda de mejores opciones profesionales/vitales. Hacen patente, por tanto, la necesidad de desarrollo personal vinculado al profesional, donde confluye la familia y el trabajo, demandando unos modelos familiares paritarios.

Las mujeres hacen referencia también a la falta de experiencia profesional con la que se enfrentan a la búsqueda de empleo, y a la necesidad de reconocer las experiencias que ellas han tenido en el ámbito laboral, es decir, de acreditación de esas experiencias y de que les cuenten dichas prácticas en los baremos que se utilizan para la selección profesional. Asimismo, en relación a la formación, las mujeres han destacado otras barreras que dificultan su reorientación profesional:

- Los bajos niveles de formación reglada, interpretados desde las escasas oportunidades que tuvieron en las etapas de escolarización y vinculado a los necesarios tempranos abandonos de la escuela.

- La escasa cualificación profesional, barrera interpretada desde la inexistencia de relaciones contractuales de trabajo y el subempleo.

- El no reconocimiento de los aprendizajes que las mujeres han acumulado con el tiempo, lo que hace que partan de cero cuando se trata de acceder al empleo.

Además de estas condiciones de partida, para las mujeres es difícil acceder a los recursos formativos y laborales, lo que se interpreta desde la escasez de medios en las zonas, así como desde las oportunidades que se le ofrecen en cada municipio. Las mujeres reflejan barreras para su mejora profesional/vital asociadas a la falta de apoyos sociales y/o locales, profesionales o para el acompañamiento al empleo, y familiares. A esto se le suman los criterios confusos en los que, en opinión de las mujeres, se basan los procesos de selección formativa y laboral en sus municipios.

Se concluye, en este sentido, que las mujeres acceden a la formación ocupacional con una escasa información profesional, que dificulta la continuidad de la misma y el paso al empleo. Quizá el que todas estas barreras sean sentidas por las mujeres podría explicar otras como las bajas expectativas de éxito profesional y la anticipación negativa a los cambios. Ellas sienten una escasa autoeficacia y baja autoestima, no reflejando confianza en sí mismas, y sintiendo que no cuentan con las posibilidades o recursos para conseguir mejorar sus trabajos.

\section{Algunas implicaciones para la orientación y formación ocupacional}

A la luz de estas conclusiones y centrándonos en algunas implicaciones, sería fundamental la eliminación de estereotipos sexistas relativos al mercado laboral, tanto en las propias mujeres y sus familias, en el empresariado y en profesionales vinculados a las acciones formativas y orientadoras. Concretamente con respecto a las mujeres es necesaria una concienciación sobre sus propias situaciones frente al empleo, de sus demandas, y de lo que estas suponen para su desarrollo profesional. Con respecto al círculo familiar y a los contextos en los que se ubican las mujeres, consideramos necesario eliminar sesgos y estereotipos sexistas pues las mujeres nos desvelan que siguen asumiendo en exclusividad las 
tareas reproductivas, las cuales afectan a sus posibilidades laborales, además de mantener percepciones sexistas sobre su rol.

Sería necesario, asimismo, aumentar el desarrollo de recursos en estas localidades, favoreciendo su difusión y utilización por parte de las mujeres, fomentar en las mujeres unas actitudes laborales positivas y acordes con características actuales del mercado laboral (por ejemplo, crear una imagen positiva hacia el cambio), pero al mismo tiempo han de ofrecerse las estructuras y recursos necesarios para la cualificación e inserción al puesto. Para ello, continuar trabajando con los mismos grupos de informantes sería especialmente interesante, porque nos permitiría implementar acciones formativas y orientadoras que les facilitasen la elaboración de sus proyectos profesionales, al mismo tiempo que dotarlas de competencias necesarias para la mejora profesional/vital.

\section{Referencias bibliográficas}

Blanch, J. M. (1992). « La transició de la mestressa de casa a la carrera per la via de l'atur”. En Actas de las IV Jornadas d'orientació sobre educació per a la carrera profesional. Barcelona. ACOEP.

Cavanaugh, J. C. y Blanchard-Fields, F. (2002). Adult development and aging. United Statesof America. Wadsworth. Thomson learning.

Varias autoras (2005-2007). Diagnóstico y autodiagnóstico de barreras de género. Posicionamiento de la mujer ante el empleo. Equal "e-Andaluzas en la Sociedad Red".

Coria, C., Freixas, A. y Covas, S. (2005). Los cambios en la vida de las mujeres. Barcelona. Paidós.

Curtis, B. (1978). “Introducción (9-41)”. En Curtis, B. y Mays, W. (Comps). Fenomenología y educación. México. Fondo de Cultura Económica.

Farmer, H. S. (1985). Model of career and achievement motivation for women and men. Journal of Counseling Psychologist, 32 (3), 363-390.

Farmer, H. S. (1997). "Women's motivation related to mastery, career salience, and career aspiration: a multivariate model focusing on the effects of sex role socialization". Journal of Career Assessment, 5 (2), 355-381.

Fitzgerald, L. F. y Weitzman, L. M. (1992). "Women's career development: theory and practice from a feminist perspective (124-160)". En H. D. Lea y Z. B. Leibowitz, Adult career development. Concepts, issues and practices. The National Career Development Association (American Association for counseling and development) ( $2^{\mathrm{a}} \mathrm{Ed}$.).

Gimeno, M. J., Rocabert, E. (1998). "Barreras percibidas por las mujeres en su proceso de toma de decisión vocacional". Revista de orientación y psicología/AEOP, 9 (15), 25-36.

Harmon, L.W. (1997). "Do gender differences necessitate separate career development theories and measures?". Journal of career assessment, 5 (4), 463-470.

Lozano, S. y Repetto, E. (2007). "Las dificultades en el proceso de decisión vocacional en relación con: el género, el curso académico y los intereses profesionales". Revista Española de Orientación y Psicopedagogía, V18, 1, 1er semestre, 5-16.

Melamed, T. (1995). "Barriers to women's career sucess: human capital, career choices, structural determinats, or simply sex discrimination". Applied psychology: an international review, 44 (4), 295-314.

Miles, M. B. y Huberman, A. M. (1994). Qualitative data analysis: an expanded sourcebook (2 $\left.{ }^{\mathrm{a}} \mathrm{Ed}.\right)$. Thounsand Oaks, California. Sage.

Montané Capdevila, J. (1993). Orientación ocupacional. Barcelona. Ceac. 
Padilla Carmona, Ma . T. (2002). "Desarrollo profesional femenino: la identidad de la mujer en el mundo laboral (73-95)". En E. Moreno y S. Villegas (Eds.), Introducción a los estudios de la mujer. Una mirada desde las Ciencias Sociales, Huelva. Diputación Provincial. Seminario de Estudios de la Mujer de la Universidad de Huelva.

Phillips, S. D. y Imhoff, A. R. (1997). "Women and career development: a decade of research". Annual review of psychology, 48, 31-59.

Poal Marcet, G. (1993). Entrar, quedarse, avanzar. Aspectos psicosociales de la relación mujermundo laboral. Madrid. Siglo XXI.

Rodríguez Gómez, G., Gil Flores, J., García Jiménez, E. (1999). Metodología de la investigación cualitativa. Archidona (Málaga). Aljibe.

Russell, J. E. A. y Burgess, J. R. D. (1998). "Success and Women's Career Adjustment". Journal of career assessment, 6 (4), 365-387.

Sánchez García, M. F. (2004). Orientación laboral. Para la diversidad y el cambio. Madrid. Sanz y Torres.

Sebastián, A., Sánchez, M. F., Sutil, M. I., Boronat, J., Cadierno, D., Solano, L. O. (2005). La presencia de estereotipos de género en el sistema educativo como determinante del desarrollo personal y profesional (Estudio descriptivo). Madrid: Dikynson.

Suárez Ortega, M. (2004). Las mujeres adultas y los procesos de reinserción laboral. Un estudio en contextos locales de la provincia de Sevilla. Diputación de Sevilla.

Suárez, Ortega, M. (En prensa). La construcción del proyecto profesional-vital de mujeres adultas: un reto para la intervención orientadora. Consejo Andaluz de Relaciones Laborales, Consejería de Empleo, Junta de Andalucía.

Swanson, J. L. y Tokan, D. M. (1991). “A college students perceptions of barriers to career development". Journal of vocational behavior, 38, 92-106.

Swanson, J. L. y Woitke, M. B. (1997). "Theory into practice in Career Assessment for women: Assessment and interventions regarding perceived carrer barriers". Journal of Career assessment, 5 (4), 443-462.

Taylor, S. J. y Bodgan, R. (1986). Introducción a los métodos cualitativos de investigación: la búsqueda de significados. Barcelona. Paidós.

Fecha de recepción 13/07/06

Fecha de revisión: 31/01/08

Fecha de aceptación: 27/02/08 